\title{
More than Just Chasing INRs: Patient-Centred Care in an Anticoagulation Clinic
}

\author{
Rosaleen Boswell and Tammy J Bungard
}

\section{INTRODUCTION}

$\mathrm{T}$ The Canadian Society of Hospital Pharmacy's 2015 initiative, targeting excellence in pharmacy practice, sets forth a vision for pharmacists to practice to their full scope and to optimize patients' medication-related outcomes by providing patientcentred care. ${ }^{1}$ Patient-centred care "is a professional obligation to take responsibility for, and provide care targeted to, the individual patient's needs". ${ }^{2}$ Fundamental elements of patientcentred care encompass respecting individual patients, appreciating their care goals and values, delivering education, enabling patients to participate in treatment decisions, and providing continuity of care. ${ }^{3}$ Within the Anticoagulation Clinic at the University of Alberta Hospital, the team's seemingly narrow focus of anticoagulation management often evolves into care issues that go beyond simply "chasing" a specific laboratory value. The purpose of this paper is to model the delivery of patient-centred care through the lens of a specialty anticoagulation clinic, where assessment of the international normalized ratio (INR) is often used as an opportunity to discover, investigate, and treat the whole patient, not just his or her lab values.

Established in 2001 with pilot project funding, the Anticoagulation Clinic is directed and staffed by 3.1 full-time equivalent clinical pharmacists, along with secretarial support, and as of spring of 2015 was managing about 750 patients. The clinic receives referrals from across Alberta for patients requiring anticoagulation therapy. Since its inception, the acceptance criteria have changed to target a more complex patient population, thus ensuring optimal resource use. ${ }^{4}$ Specifically, patients accepted are those with high thromboembolic risk, high risk for major hemorrhage, complex comorbidities, or medications that affect anticoagulation control. This higher-risk patient population requires that pharmacists collaborate with other practitioners across the health care continuum and use their additional prescribing authority to proactively change warfarin dosing and anticoagulant therapy to ensure safe and effective care delivery. Despite practising in a very specialized setting, pharmacists in the Anticoagulation Clinic use comprehensive patient assessment and investigational skills to manage these complex cases, often making interventions in areas not directly related to anticoagulation therapy.

This paper presents several case vignettes to describe the scope of pharmacists' practice at the Anticoagulation Clinic. ${ }^{*}$ In particular, we reflect on the evolution of skill sets that now extend beyond the narrow, misperceived goal of simply "chasing an INR between 2.0 and 3.0 ", in alignment with the provision of patient-centred care.

\section{Working hard and working smart sometimes can be two different things. -Byron Dorgan, US politician (1942- )}

Case vignette 1: An inpatient was referred to the Anticoagulation Clinic after experiencing an incidental pulmonary embolism following a motor vehicle crash. The surgeon had consulted a hematologist, and the patient was subsequently kept in hospital on a course of low-molecular-weight heparin (LMWH) during transition to warfarin for a 3-month course of therapy (the patient could not afford the LMWH therapy, which would have had to be purchased after discharge). At the time of intake to the Anticoagulation Clinic, a discussion of all therapy options with the patient revealed lack of acceptance of both LMWH (because of the cost) and warfarin (because of the need for ongoing INR testing). The patient was unaware of the intended 3-month duration of therapy (i.e., therapy would not continue indefinitely) and, once the total cost of therapy had been reviewed, opted to purchase rivaroxaban. The pharmacist in the Anticoagulation Clinic closed the loop by paging the specialist physicians, writing a discharge prescription for rivaroxaban, and facilitating discharge from hospital the same day.

\footnotetext{
*The case vignettes are based on real cases from the Anticoagulation Clinic. Patient consent was not obtained; however, potentially identifying information not important to the message of the paper has been removed. The quotations used as headings in this article were derived from www.braineyquote.com
} 
Referrals to the Anticoagulation Clinic may come from any institution (for inpatients) or from family physicians, specialists, or other health care professionals (for outpatients). Referrals are received by fax or phone, and then undergo initial assessment and triage by a pharmacist. This process encompasses collating information from various sources, including the provincial electronic health record (Alberta Netcare), clinic letters from specialists, and direct conversations with the referring practitioner and patient. When an outpatient referral is accepted, an appointment is made to conduct an in-person initial visit. This ensures that the patient is made aware of the role of the Anticoagulation Clinic and his or her own responsibilities and allows collection of a detailed history and provision of individualized education. For inpatient referrals at the University of Alberta Hospital, as in this case, a pharmacist often goes to the patient's bedside before discharge to complete this step in a timely manner.

This case demonstrates the ability of the Anticoagulation Clinic to provide patient-centred care by collating all pertinent patient information (medical and medication history, patient's knowledge, beliefs, and values) alongside the impressions and diagnostic information available from the patient chart and health care team. Despite the patient's already having been assessed by specialist teams and referred to the Anticoagulation Clinic for ongoing warfarin management, clinic staff assessed the appropriateness of the current therapy and explored all therapeutic alternatives with the patient. The ability to intervene with an alternative to LMWH or warfarin demonstrates individualized patient care and cost saving to the health care system.

\section{The single biggest problem in communication is the illusion that it has taken place. -George Bernard Shaw, author (1856-1950)}

Case vignette 2: A high-risk patient, who was to be discharged with dual-antiplatelet and warfarin therapy, was accepted by the Anticoagulation Clinic for outpatient management. Despite having communicated with the hospital unit, the clinic was not notified at the time of discharge, nor was any information about the discharge sent to the clinic. The clinic discovered that discharge had occurred, intervened on the patient's discharge plan (reducing the advised warfarin dose by $50 \%$ ), and sent the patient for INR testing 2 days earlier than hospital staff had instructed. The INR result was 4.3 , in a patient already at high risk for major hemorrhage.

The Anticoagulation Clinic has an electronic database with a function for tracking patients expected to be discharged from hospital. This allows for follow-up if, as in this case, communication about the discharge does not occur. Having a systematic approach to ensure that patients do not "fall through the cracks" allows clinic pharmacists to provide responsive and timely care. Aside from having the clinical skill set to deliver care, success in care delivery stems from being responsive and accountable to patients and, ultimately, having sufficient resources to follow through on matters in a timely fashion. Furthermore, the ability of clinic pharmacists to use additional prescriptive authority to change prescriptions and take ownership of such decisions allows for proactive care. ${ }^{6}$

\section{A smart man makes a mistake, learns from it, and never makes that mistake again. But a wise man finds a smart man and learns from him how to avoid the mistake altogether. -Roy H Williams, author (1958- )}

Case vignette 3: A cardiologist on the hospital ward called the Anticoagulation Clinic after being paged about an INR above 9.0 for a patient discharged a week previously, now back living in a rural community. The clinic had originally declined this referral because of the patient's low thromboembolic risk and the availability of the family doctor for follow-up. The cardiologist requested assistance in dealing with the critical INR result. Clinic staff agreed, contacted the patient, and confirmed that no bleeding had occurred and that there was no need for urgent care referral. Further assessment showed that the patient had diarrhea soon after discharge (which likely contributed to the critical INR), and stool cultures were positive for Clostridium difficile. Investigation of drug therapies revealed that the family physician had originally prescribed the correct antibiotic to treat $C$. difficile, namely metronidazole. However, the community pharmacist had intervened because of the known major drug interaction of metronidazole with warfarin, and obtained approval from the family doctor to change the antibiotic to clindamycin, an inappropriate choice for treating C. difficile. The Anticoagulation Clinic collaborated with the patient, the family doctor, and the community pharmacist to effectively manage this critical INR (by holding the warfarin and prescribing oral vitamin $\mathrm{K}$ ) and to provide education about the cause of the critical INR (unresolved diarrhea). The Anticoagulation Clinic assumed ongoing warfarin management for this patient and also ensured appropriate management of the $C$. difficile infection (with metronidazole), using strategies to manage the drug interaction between metronidazole and warfarin.

With limited specialty clinic resources and funding, targeting higher-risk patients is logical at a conceptual level, because the greatest impact in care should be evident in those needing it most. On the surface, this case appeared straightforward, and the patient did not meet the criteria for acceptance into the Anticoagulation Clinic. However, management in the rural community was poor, which elevated the patient's risk level. Used properly, anticoagulants are life-saving therapies; however, the risk of misadventure may yield deadly consequences. This case raises the question of whether an anticoagulation clinic should accept all patients for whom warfarin is prescribed, given the potential for negative outcomes if such therapy is not properly managed. 
Moreover, this case highlights ongoing collaboration of the Anticoagulation Clinic both within the tertiary care setting (with the cardiologist) and in the community (with the family doctor and community pharmacist) as we endeavoured to ensure appropriate management of $C$. difficile and, in turn, the warfarin therapy. This case supports the concept of pharmacists prescribing all regulated provincial drugs, as the same process is used across various disease states to identify a drug-related problem and take steps to resolve it.

\section{It's not that I'm so smart, it's just that I stay with problems longer. -Albert Einstein, physicist (1879-1955)}

Case vignette 4: During routine follow-up with an older patient who had high thromboembolic risk (because of a history of mechanical mitral valve replacement), the Anticoagulation Clinic noted alterations in the patient's speech alongside a reported history of headaches over the previous 2 weeks. The family attributed these problems to advanced age and a recent diagnosis of anemia, but staff in the Anticoagulation Clinic were more concerned and referred the patient to the nearest emergency department. Following up the next day, the Anticoagulation Clinic found that the patient had been transferred from the local (rural) emergency department to an urban tertiary care centre, had undergone head computed tomography (CT), and had been discharged home with no instructions or guidance. The clinic pharmacist reviewed the investigations, and found that bilateral chronic subdural hematomas had been noted on the CT report, along with a drop in hemoglobin. The patient was unaware of these results. The Anticoagulation Clinic contacted a neurologist, who also reviewed the CT scan and insisted that the patient return immediately for re-assessment. The antithrombotic therapy was modified, to enable healing of the subdural hematomas, with long-term alteration of the INR target.

At the Anticoagulation Clinic, follow-up is typically performed by telephone. Laboratory test results (such as INR) are reviewed, and changes to health, medications, lifestyle, signs and symptoms of clotting or bleeding, and upcoming medical procedures and appointments are discussed. A plan is implemented for anticoagulant dosing, and future follow-up is scheduled. Given the constancy of patient contact, staff in the Anticoagulation Clinic develop relationships with patients and their caregivers, and this case highlights the ability to effectively assess and detect important changes with an individual patient even when family members are less concerned. Our ability to consult specialist physicians for insight and assistance provides the infrastructure that allows clinic pharmacists to continue to investigate and manage complex cases. These clinically based inquiries rely on having the insight to discover, appropriately investigate, and make an effort to resolve any issues-akin to taking that extra step in patient-centred care. As the scope of pharmacists' practice has evolved to include the assessment and supportive care required to assume full ownership of drug prescribing and monitoring decisions, it is paramount that workload systems capture both the time allocated and the importance of these ancillary responsibilities.

\section{It's not what you look at that matters, it's what you see.}

\section{—Henry David Thoreau, author (1817-1862)}

Case vignette 5: An older patient with atrial fibrillation $\left(\mathrm{CHADS}_{2}\right.$ score $=2$, with left ventricular dysfunction and diabetes mellitus; taking acetylsalicylic acid $81 \mathrm{mg}$ daily) was switched from warfarin to rivaroxaban by the Anticoagulation Clinic. During routine follow-up, it was noted that the hemoglobin had dropped from $120 \mathrm{~g} / \mathrm{L}$ at baseline to $73 \mathrm{~g} / \mathrm{L}$. The patient denied signs or symptoms of bleeding, but clinic staff advised going to the emergency department anyway. The patient was admitted to hospital and received a transfusion. Outpatient colonoscopy was arranged, and in the interim the rivaroxaban was held. Proper colonoscopy preparation was not performed, which led to cancellation of the procedure, with the next available date 2 months away. Acknowledging the urgency of this assessment, the clinic advocated for more rapid rescheduling of the colonoscopy, which ultimately took place 2 days later. A large bleeding colonic polyp was discovered and removed. The Anticoagulation Clinic pharmacist contacted the cardiologist to confirm discontinuation of the antiplatelet therapy, to minimize the risk of future bleeding, and restarted rivaroxaban in a timely fashion.

Ongoing follow-up integrates the interpretation of laboratory values in light of a patient's health history and reported signs and symptoms. In this case, despite the patient's report of being clinically well, the degree of decline in hemoglobin was very concerning and prompted referral. Given the patient's thromboembolic risk, a prolonged delay in anticoagulant therapy was inappropriate yet necessary until the source of the bleeding could be identified and managed. The Anticoagulation Clinic pharmacist made multiple telephone calls to coordinate timely and appropriate care for this patient. This type of workload, related to navigating the health care system, is very time-consuming. To this end, workload capture is important to ensure that resources are allocated correctly and time-tracking is accurate, given the clinic's fixed resources and inability to accept all new referrals.

\section{Obstacles are those frightful things you see when you take your eyes off your goal. -Henry Ford, businessman (1863-1947)}

Case vignette 6: During routine follow-up for a patient with a mechanical mitral valve who was receiving warfarin therapy, the patient asked whether "it was still safe to proceed" with a 
dental appointment the following day. Clinic staff confirmed that it was safe to proceed, given the current INR. However, further investigation revealed no intention (on the part of the dental office) to prescribe antibiotic prophylaxis, nor was there any history of prophylactic therapy. After communication between the clinic and the dental office, the dentist requested a written letter from the Anticoagulation Clinic, outlining the necessity of antibiotic prophylaxis for this patient.

This case highlights a simple example of how a narrowly based question related to the INR evolved into ensuring appropriate patient-centred care. Other examples frequently encountered in the Anticoagulation Clinic include ensuring that inferior venal caval filters are removed, that duration of anticoagulation therapy is reassessed, and that patients with mechanical heart valves have ongoing cardiology follow-up. As pharmacists's scopes of practice evolve to include taking ownership and responsibility for drug-related decisions, their responsibility to appropriately assess and act on other clinical issues that are identified must develop in alignment with expectations for proactive management.

\section{CONCLUSIONS}

The seemingly narrow task of the Anticoagulation Clinic to "chase that INR" of 2.0-3.0 has been embraced and expanded to ensure optimal patient care delivery. In optimizing patient care, the Anticoagulation Clinic Team must assess the therapy at hand, yet also probe deeper when necessary, knowing when to ask for more details and which areas to build on. As a result, team members must often deal with clinical concerns that are not directly related to anticoagulant therapy, yet are relevant to providing patient-centred care. Through the case vignettes presented here, we have highlighted many of the challenges to optimal delivery of patient-centred care, including deficiencies in front-line clinicians' foundational knowledge, lack of patient education and hence lack of patient engagement in the decisionmaking process, and limited safeguards within the healthcare system to ensure appropriate and timely follow-up care. To ensure effectiveness in their activities, pharmacists in the Anticoagulation Clinic have a foundational clinical knowledge base (one that goes beyond "chasing INRs"), systems to ensure accountability in proactive care delivery, and a working overview of the health care system. As the scope of pharmacist practice has evolved, with authorization to prescribe provincially regulated drugs and to order laboratory tests, pharmacists must accept further responsibilities necessary to fulfill their role, such as communication (written and verbal) with other providers, on-call duties to receive critical laboratory results, and all associated paperwork. It is also important that workload-tracking systems evolve to capture human resource time allocation and the daily activities of this evolving role. These changes in workload tracking will justify the sustainability of pharmacists' current role in delivering patientcentred care and will also document an awareness of ongoing role evolution.
We have used our specialty Anticoagulation Clinic as an example of how patient-centred care can be delivered and would suggest that the concepts described herein are applicable to any practice area. Appropriately assessing the INR represents an opportunity within our practice setting to provide patientcentred care, and we encourage all pharmacists to explore and utilize similar opportunities or triggers for such care in their own practice areas. As a profession, we must embrace change, move outside our comfort zones to gain new experiences, and welcome life-long learning to provide the best patient care possible.

\section{The pessimist complains about the wind; the optimist expects it to change; the realist adjusts the sails. —William Arthur Ward, author (1921-1994)}

\section{References}

1. CSHP vision 2015. Ottawa (ON): Canadian Society of Hospital Pharmacists; [cited 2014 Aug 26]. Available from: www.cshp.ca/cshp2015/

2. Epstein RM, Street RL Jr. The values and value of patient-centered care. Ann Fam Med. 2011;9(2):100-3.

3. Tsuyuki RT, Krass I. What is patient-centred care? Can Pharm J. 2013; 146(4):177-80.

4. Bungard TJ, Archer SL, Hamilton P, Ritchie B, Tymchak W, Tsuyuki RT. Bringing the benefits of anticoagulation management services to the community: Alberta program may serve as a model of care. Can Pharm J. 2006;139(2): 58-63.

5. Garcia DA, Witt DM, Hylek E, Wittkowsky AK, Nutescu EA, Jacobson A, et al. Delivery of optimized anticoagulant therapy: consensus statement from the Anticoagulation Forum. Ann Pharmacother. 2008;42(7):979-88.

6. Yuksel N, Eberhart G, Bungard TJ. Prescribing by pharmacists in Alberta. Am J Health Syst Pharm. 2008;65(22):2126-32.

Rosaleen Boswell, BScPharm, ACPR, is a clinical hospital pharmacist with Alberta Health Services, University of Alberta Hospital, Edmonton, Alberta.

Tammy J Bungard, BSP, PharmD, is Director of the Anticoagulation Management Service, University of Alberta Hospital, and an Associate Professor in the Division of Cardiology, Department of Medicine, University of Alberta, Edmonton, Alberta.

Competing interests: Tammy J Bungard has received honoraria from Bayer, BMS-Pfizer, and Boehringer-Ingelheim for activities outside the topic of this article. No competing interests declared by Rosaleen Boswell.

\section{Address correspondence to:}

Dr Tammy J Bungard

Research Transition Facility

EPICORE Centre

University of Alberta

4000, 8308-114 Street

Edmonton $A B$ T6G 2V2

e-mail: tammy.bungard@ualberta.ca

Acknowledgements: The authors would like to acknowledge the entire Anticoagulation Clinic Team (Norelle Evanger, Angela Gee, Rhonda Lemoine, Karen Schultz, and Jodi Wilkie) for their dedication to clinic patients and their desire to always take that extra step to ensure delivery of quality care across the health care spectrum. The authors would also like to thank Dr. Ross Tsuyuki for his critical review, as well as insight and assistance in framing this manuscript. 